\title{
Parallel Interleaver Design for a High Throughput HSPA+/LTE Multi-Standard Turbo Decoder
}

\author{
Guohui Wang, Student Member, IEEE, Hao Shen, Yang Sun, Member, IEEE, Joseph R. Cavallaro, Senior \\ Member, IEEE, Aida Vosoughi, Student Member, IEEE, and Yuanbin Guo, Senior Member, IEEE
}

\begin{abstract}
To meet the evolving data rate requirements of emerging wireless communication technologies, many parallel architectures have been proposed to implement high throughput turbo decoders. However, concurrent memory reading/writing in parallel turbo decoding architectures leads to severe memory conflict problem, which has become a major bottleneck for high throughput turbo decoders. In this paper, we propose a flexible and efficient VLSI architecture to solve the memory conflict problem for highly parallel turbo decoders targeting multistandard 3G/4G wireless communication systems. To demonstrate the effectiveness of the proposed parallel interleaver architecture, we implemented an HSPA+/LTE/LTE-Advanced multi-standard turbo decoder with a 45nm CMOS technology. The implemented turbo decoder consists of 16 Radix-4 MAP decoder cores, and the chip core area is $2.43 \mathrm{~mm}^{2}$. When clocked at $600 \mathrm{MHz}$, this turbo decoder can achieve a maximum decoding throughput of $826 \mathrm{Mbps}$ in the HSPA+ mode and $1.67 \mathrm{Gbps}$ in the LTE/LTEAdvanced mode, exceeding the peak data rate requirements for both standards.
\end{abstract}

Index Terms-Turbo decoder, interleaver, parallel processing, VLSI architecture, ASIC implementation, memory contention, HSPA+, LTE/LTE-Advanced.

\section{INTRODUCTION}

D URING the past few years, modern 3G/4G wireless communication systems such as 3GPP (3rd Generation Partnership Project) UMTS/HSPA+ (Universal Mobile Telecommunications System/High-Speed Packet Access Evolution) [2], 3GPP LTE (Long Term Evolution) and LTEAdvanced [3] have been deployed to meet the ever-growing demand for higher data rates and better quality of service. High throughput is one of the most important requirements for emerging wireless communication standards. For instance, the 3GPP UMTS standard Release 11 extends HSPA+ with several key enhancements including increased bandwidth and number of antennas. These enhancements lead to $336 \mathrm{Mbps}$

Manuscript received September 14, 2013; revised November 28, 2013, February 01, 2014; accepted February 02, 2014. This paper was presented in part at the IEEE International Symposium on Circuits and Systems (ISCAS), Beijing, China, May 2013 [1]. This work was supported in part by Huawei, and by the US National Science Foundation under grants CNS-1265332, ECCS-1232274, and EECS-0925942.

G. Wang, H. Shen, Y. Sun, J. R. Cavallaro and A. Vosoughi are with the Department of Electrical and Computer Engineering, Rice University, Houston, TX 77005, USA (email: wgh@ @rice.edu). Y. Guo is with Wireless R\&D, US Research Center, Futurewei Technologies, 5340 Legacy Dr., Plano, Texas 75024, USA.

Copyright (c) 2014 IEEE. Personal use of this material is permitted. However, permission to use this material for any other purposes must be obtained from the IEEE by sending an email to pubs-permissions@ieee.org.

Digital Object Identifier 10.1109/TCSI.2014.2309810 peak data rate with $2 \times 2$ MIMO (multiple-input multipleoutput) and $40 \mathrm{MHz}$ bandwidth (or $4 \times 4 \mathrm{MIMO}$ and $20 \mathrm{MHz}$ bandwidth) [2]. Recently, up to $672 \mathrm{Mbps}$ data rate has been proposed for the future release of 3GPP standards [4], [5]. As a $4 \mathrm{G}$ candidate, the $3 \mathrm{GPP}$ LTE-Advanced promises up to 1 Gbps data rate as its long term goal.

Turbo codes are specified in many wireless communication standards such as the HSPA+ and LTE/LTE-Advanced as forward error-correction codes to ensure reliable communications via wireless channels, due to their outstanding error-correcting performance [6]. A turbo decoder contains two key components: soft-input soft-output (SISO) decoders and interleavers. During the decoding process, log-likelihood ratio (LLR) soft values are exchanged between component SISO decoders in an iterative way. The interleaver is a critical component for the turbo decoder to achieve good error-correcting performance, by permuting the LLRs randomly between iterations and maximizing the effective free distance of turbo codes. Since the introduction of turbo codes, numerous VLSI architectures and implementations have been proposed [7]-[10]. To achieve high throughput, parallel turbo decoding architectures are usually employed, in which several SISO decoders operate in parallel with each working on a segment of the received codeword [9][24]. The parallel turbo decoders suffer from severe memory conflict problems due to the randomness of the interleaver, which becomes a major challenge for high throughput designs and implementations [11], [15], [16], [20], [21], [25]-[36].

Although LTE and LTE-Advanced can provide higher data rates than HSPA+, the high cost of LTE infrastructure prevents the rapid deployment of LTE systems; in contrast, existing infrastructure can be upgraded with low cost to support the new features defined in HSPA+. Therefore, HSPA+ and LTE are expected to co-exist for a long term, especially when HSPA+ still keeps evolving rapidly [4], [5]. It is of great interest for mobile devices to support multiple standards. As an essential building block, multi-standard turbo decoders have been studied in the literature [10], [13], [14], [16], [18], [20], [23], [34], [37]-[39]. However, since the challenging memory conflict problem caused by the HSPA+ interleaving algorithm limits the parallelism degree of turbo decoders, none of them can meet the high throughput requirements of the recent HSPA+ extensions (336 Mbps for 3GPP Release 11 and 672 Mbps proposed for 3GPP Release 12 and up).

In this paper, we propose a parallel interleaving architecture to solve the memory conflict problem for highly parallel multi-standard turbo decoding. The remainder of the paper is organized as follows. Section III introduces the memory 


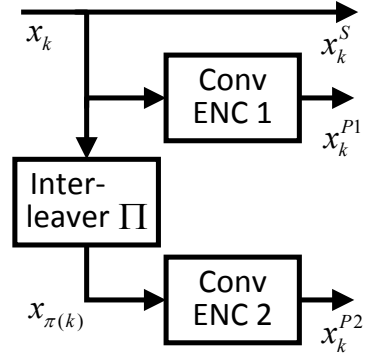

Turbo encoder

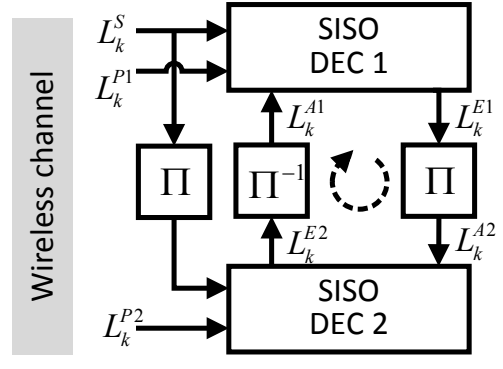

Turbo decoder
Fig. 1. Block diagram for a turbo encoder (left) and a turbo decoder (right).

conflict problem in parallel turbo decoding and related work focusing on the solutions to solve the memory conflict problem. In Section [III, we describe a balanced turbo decoding scheduling scheme to eliminate memory reading conflicts. In Section IV] we present a double-buffer contention-free (DBCF) buffer architecture to solve the memory writing conflict problem. In Section $\mathrm{V}$, we adapt a unified and on-thefly interleaver/deinterleaver address generation architecture to the multi-standard turbo decoding architecture. In Section VI we present a high throughput implementation of HSPA+/LTE multi-standard turbo decoder to demonstrate the effectiveness of the proposed architecture. Finally, we conclude this paper in Section VII.

\section{BACKGROUND AND CHALlENGES}

The turbo code encoder specified in HSPA+ and LTE standards consists of two 8-state component convolutional encoders and an interleaver. As is shown on the left hand side of Fig 1 , when a block of information bits $x_{k}(k=0,1, \ldots, K-1)$ is streamed into an encoder, a sequence of systematic bits $x_{k}^{S}\left(x_{k}^{S}=x_{k}\right)$, a sequence of parity-check bits $x_{k}^{P 1}$ and a second sequence of parity-check bits $x_{k}^{P 2}$ are generated and transmitted over the wireless channel. The interleaver permutes the input bits $x_{k}$ to $x_{\pi(k)}$ based on the interleaving law $\pi$ defined in standards.

\section{A. Turbo Decoding Algorithm}

A turbo decoder receives soft reliability information for the transmitted sequences $x_{k}^{S}, x_{k}^{P 1}$ and $x_{k}^{P 2}$, in the form of log-likelihood ratios (LLRs), denoted as $L_{k}^{S}, L_{k}^{P 1}$ and $L_{k}^{P 2}$, respectively. The LLR indicates the probability of a received bit being a binary 0 or 1 . Shown in the right hand side of Fig. 1. a turbo decoder consists of two constituent softinput soft-output (SISO) decoders and an interleaver. The idea of the turbo decoding algorithm is to iteratively update the probability information between two SISO decoders, separated by an interleaver and a deinterleaver. In one iteration, two SISO decoders process reliability information and exchange so-called extrinsic information. The computation of each SISO decoder is called one half-iteration. In the first half-iteration, SISO decoder 1 computes extrinsic information $L_{k}^{E 1}$ using the LLRs of the received bits $L_{k}^{S}$, parity-check bits $L_{k}^{P 1}$ and a priori information $L_{k}^{A 1} . L_{k}^{A 1}$ is generated by deinterleaving the extrinsic information $L_{k}^{E 2}$ from the other SISO decoder

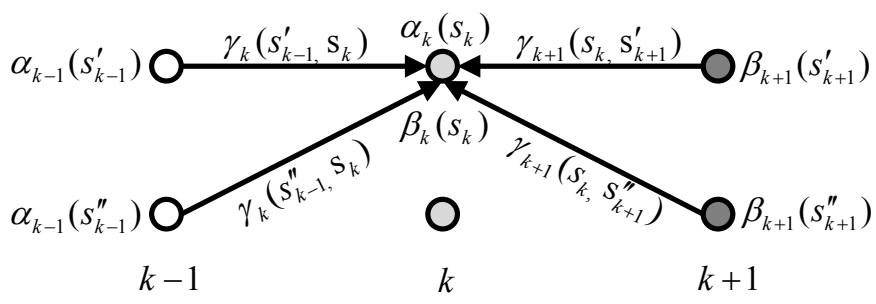

Fig. 2. Forward and backward recursions for trellis step $k$ in the MAP decoding algorithm.

$\left(L_{k}^{A 1}=L_{\pi^{-1}(k)}^{E 2}\right)$. Similarly, during the second half-iteration, SISO decoder 2 generates extrinsic information $L_{k}^{E 2}$ based on $L_{\pi(k)}^{S}, L_{k}^{P 2}$ and $L_{k}^{A 2}\left(L_{k}^{A 2}=L_{\pi(k)}^{E 1}\right)$. This iterative process continues until a preset maximum number of iterations is reached or a stopping criterion is met.

The maximum a posteriori (MAP) algorithm is normally used to implement SISO decoders [6]. The MAP decoding process can be represented as a trellis traversal. The trellis nodes represent the states of convolutional codes, and the branches represent state transitions. Branch metric $\gamma_{k}(s, t)$ denotes a transition from state $s$ to state $t$ at step $k$. As shown in Fig. 2. the trellis traversal is performed in both forward and backward directions to compute the state metrics $\alpha_{k}\left(s_{k}\right)$ and $\beta_{k}\left(s_{k}\right)$ for all eight states:

$$
\begin{aligned}
\alpha_{k}\left(s_{k}\right)= & \stackrel{*}{*}\left\{\alpha_{k-1}\left(s_{k-1}^{\prime}\right)+\gamma_{k}\left(s_{k-1}^{\prime}, s_{k}\right),\right. \\
& \left.\alpha_{k-1}\left(s_{k-1}^{\prime \prime}\right)+\gamma_{k}\left(s_{k-1}^{\prime \prime}, s_{k}\right)\right\}, \\
\beta_{k}\left(s_{k}\right)= & \max \left\{\beta_{k+1}\left(s_{k+1}^{\prime}\right)+\gamma_{k+1}\left(s_{k}, s_{k+1}^{\prime}\right),\right. \\
& \left.\beta_{k+1}\left(s_{k+1}^{\prime \prime}\right)+\gamma_{k+1}\left(s_{k}, s_{k+1}^{\prime \prime}\right)\right\},
\end{aligned}
$$

where the max operation is typically implemented using the max-log approximation as follows:

$$
\stackrel{*}{*}^{*}(x, y)=\max (x, y)+\log \left(1+e^{-|x-y|}\right) \approx \max (x, y) .
$$

Once the forward metrics and backward metrics are computed, the LLRs of the a posteriori probabilities (APPs) for information bits $x_{k}$ are computed as follows:

$$
\begin{gathered}
L\left(\hat{x}_{k}\right)=\max _{\left(s_{k-1}, s_{k}\right): x_{k}=0}^{*}\left\{\alpha_{k-1}\left(s_{k-1}\right)+\gamma_{k}\left(s_{k-1}, s_{k}\right)+\beta_{k}\left(s_{k}\right)\right\} \\
-\underset{\left(s_{k-1}, s_{k}\right): x_{k}=1}{*}\left\{\alpha_{k-1}\left(s_{k-1}\right)+\gamma_{k}\left(s_{k-1}, s_{k}\right)+\beta_{k}\left(s_{k}\right)\right\} .
\end{gathered}
$$

For each MAP decoder $i(i \in\{1,2\})$, the extrinsic LLR information is computed by $L_{k}^{E i}=L\left(\hat{x}_{k}\right)-L_{k}^{S}-L_{k}^{A i}$.

\section{B. Parallel Turbo Decoding Architecture}

To achieve high throughput, numerous parallel turbo decoding architectures have been extensively investigated and several levels of parallelisms have been explored [1], [10][12], [14]-[17], [19]-[21], [23].

Most parallel turbo decoding algorithms exploit parallelism in SISO-decoder level, where a codeword (with block size $K)$ is partitioned into $P$ sub-blocks with size of each being $K / P$. Multiple SISO decoders work in parallel with each operating on one of the sub-blocks as shown in Fig. 3 . The 


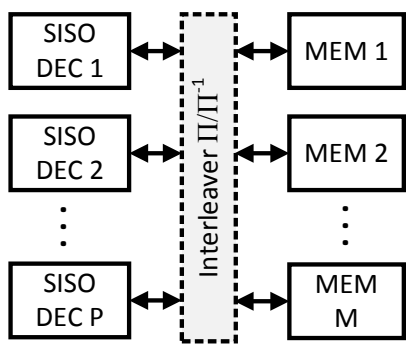

(a)

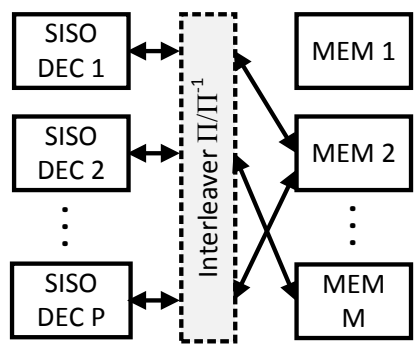

(b)
Fig. 3. (a) Parallel turbo decoding architecture with $P$ SISO decoders and $M$ extrinsic memory modules; (b) A 2-way memory conflict happens when SISO decoder 1 and SISO decoder $P$ access (read from or write to) memory 2 at the same time.

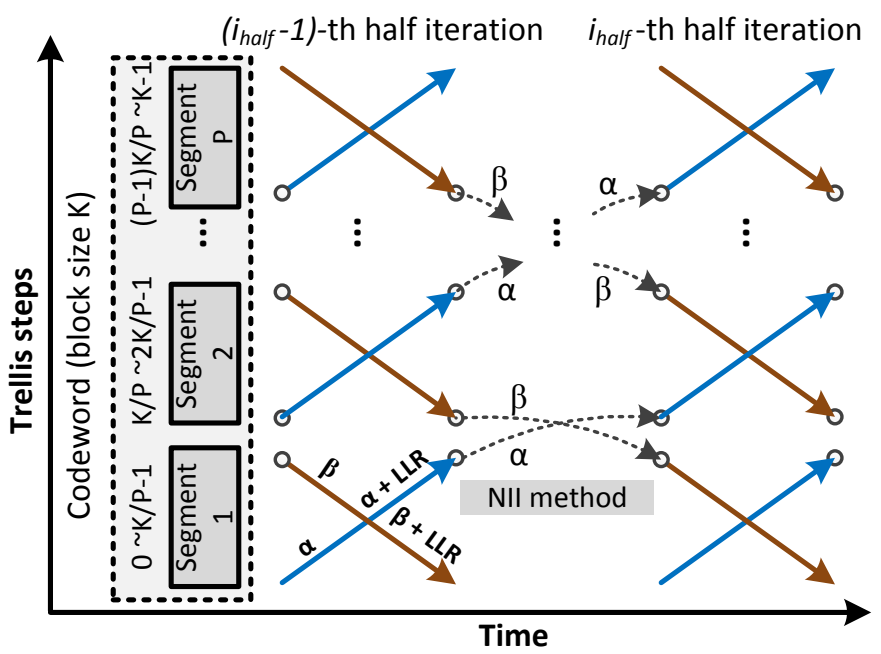

Fig. 4. Diagram of the cross-MAP (XMAP) decoding architecture with NII.

Radix-4 SISO decoding algorithm is another way to increase the throughput by exploring the trellis-level parallelism. It applies a one-step look-ahead concept to the forward/backward trellis recursions, in which two trellis steps are computed simultaneously [15], [17], [19], [20], [38]. In addition to the above algorithms, many variants of SISO decoder architectures explore parallelism from other aspects such as recursion-unit level parallelism, sliding window schemes and so on [10], [14], [15], [17], [19], [20]. Therefore, it is quite challenging to design an interleaver architecture which is able to handle the aforementioned different variants of parallel turbo decoding algorithms. Our goal is to design a flexible and scalable interleaver architecture which can fulfill this requirement.

In our implemented parallel turbo decoder, we choose a Radix-4 cross-MAP (namely XMAP) decoding architecture to implement the SISO decoders due to its high throughput performance [19]. In an XMAP decoding architecture as is shown in Fig. 4, $\alpha$ and $\beta$ recursion units work in parallel in a crossed manner, towards the forward and backward directions, respectively. In each half iteration, before the cross point, the $\alpha$ and $\beta$ recursion units compute $\alpha$ and $\beta$ state metrics. After the cross point, each Radix-4 XMAP decoder starts to produce four extrinsic LLR values per clock cycle. When $P$ such SISO decoders working in parallel to decode a codeword with block size $K$, each SISO decoder performs computation on a segment (or sub-block) of the codeword with size $K / P$. A method call Next Iteration Initialization (NII) is employed to avoid acquisitions for the $\alpha$ and $\beta$ state metrics [8], [19]. Only the stake information in the end of the recursion in a sub-block is saved and propagated between SISO decoders.

\section{Design Challenges}

A parallel turbo decoder which generates $P_{L L R}$ extrinsic LLRs in each clock cycle requires a memory bandwidth of $P_{L L R}$ memory accesses per clock cycle. Memory used to store the extrinsic LLR values are partitioned into $M\left(M \geq P_{L L R}\right)$ memory modules to provide higher memory bandwidth. However, due to the randomness of the interleaver/deinterleaver, multiple SISO decoders may attempt to access the same memory bank concurrently, which causes a memory conflict, as is depicted in Fig. 3p. A significant reduction in effective memory bandwidth caused by frequent memory conflicts decreases the decoding throughput. Therefore, the memory conflict problem has become a major bottleneck in the design of a high throughput parallel turbo decoder [1], [11], [15], [16], [20], [21], [25]-[35].

A traditional way of implementing the turbo code interleaver is static memory mapping when the parallelism of turbo decoders is low. The interleaving patterns are precomputed and stored in a look-up table (LUT). For every decoding algorithm and parallel architecture, a unique LUT is required since significantly different memory access patterns are generated. If multiple standards and variable block sizes need to be supported, memory usage for LUTs will grow drastically. This apparently is not efficient for an embedded SoC implementation. Therefore, on-the-fly interleaver address generation (IAG) is preferred in highly parallel turbo decoders since it requires less memory and can be easily parametrized to support different configurations and multiple standards.

\section{Related Work}

Many interleaver architectures have been proposed to solve the memory conflict problem in parallel turbo decoding systems, and they can be classified into three categories: designtime solutions, compilation-stage solutions and runtime solutions [10], [33].

(1) Design-time solutions usually employ algorithmarchitecture co-design methods in the early design stage [15], [33]. Contention-free algorithms are developed by applying specific algebraic properties to the interleaver algorithms. As examples, the ARP (almost regular permutation) interleaver adopted by the WiMax standard and the QPP (quadratic permutation polynomial) interleaver used by LTE/LTE-Advanced standards fall into this category [29], [31]. However, interleavers designed by these design-time methods can be contention-free only under some constraints. For instance, the QPP interleaver is contention-free when the decoding parallelism is a factor of the block size $K$ [19], [31]. In addition, these solutions lack flexibility to support existing interleaving algorithms, such as the 3GPP HSPA+ interleaver. Therefore, in any of the above cases, we should seek other solutions. 


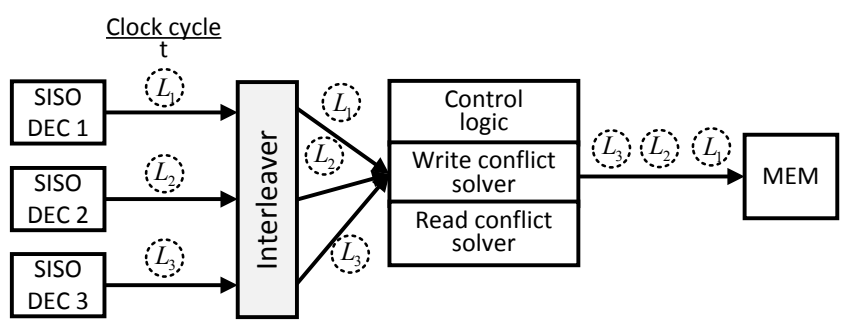

(a)

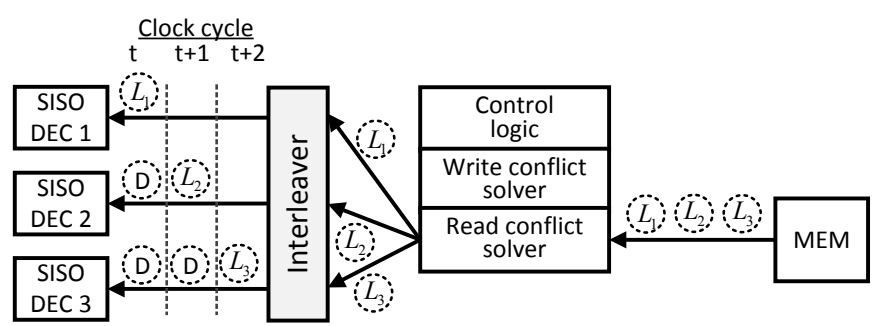

(b)

Fig. 5. (a) Memory writing conflict, in which 3 SISO decoders try to write to the same memory; (b) Memory reading conflict, in which 3 SISO decoders try to read data from the same memory. $L_{1}, L_{2}$ and $L_{3}$ represent LLR data; $D$ represents a clock cycle's delay.

(2) Compilation-stage solutions employ certain memory mapping rules to generate data access patterns to avoid memory conflicts [12], [21], [25], [28], [35]. It has been shown by Tarable et al. that there is always a memory mapping scheme which allows contention-free memory accessing for any interleaving algorithm in a parallel turbo decoder [28]. Recently, Chavet et al. proposed memory mapping methods based on graph coloring algorithms, in which a memory mapping pattern can be calculated in polynomial time [21], [35]. The limitation of the compilation-stage solutions is that they require complex off-line computations to generate memory mappings and a large amount of memory resources to store memory mappings. To alleviate this problem, Ilnseher et al. proposed to compress permutation vectors to reduce the memory requirement. However, the permutation vectors still lead to large memory area, even when a hybrid compression method is used [10].

(3) Runtime conflict solutions use buffers or on-chip networks to dynamically reschedule data access orders to avoid memory conflicts [11], [16], [20], [26], [27], [30], [32], [34], [36]. As discussed above, both the design-time solution and compilation-stage solution require the memory access patterns to be precomputed and stored in memory. As a comparison, a runtime conflict solution does not need large memories. It can provide a maximum degree of flexibility, enabling highly configurable multi-standard turbo decoder implementation. Therefore, we prefer runtime conflict resolution. In the rest of this section, some existing runtime solutions are briefly described.

Thul et al. proposed a tree-based concurrent interleaving architecture (namely TIBB) and an improved solution based on local buffer cells interconnected via a ring network (namely RIBB), respectively [11], [26]. These solutions have a high connectivity of LLR distributors, which limit the max clock frequency of the circuits and the parallelism of a turbo decoder. In [27], Speziali et al. extended Thul's work and proposed an improved architecture for the interconnection network. A stalling mechanism is introduced to the interleaver architecture. However, the stalling mechanism leads to a high hardware complexity and results in unacceptable delay penalty for highly parallel SISO decoder architectures such as Radix-4 SISO decoders. Recently, Network-on-chip (NoC) approaches have been proposed to solve the memory conflict problem. For example, in [30], [32], [36], the authors proposed packetswitched NoC approaches such as Butterfly NoC and Benesbased NoC architectures. However, these NoC methods suffer from a large delay penalty limiting the maximum throughput. Furthermore, to avoid network contention, complex scheduling methods and control logic are required to schedule the network packets. In [20], Asghar et al. presented misalignment among memory access paths using delay line buffers. In fact, these schemes can only alleviate the memory conflict problem when the parallelism of turbo decoder is low. The delay line buffers also cause long delay and increase hardware cost.

To overcome the limitations of the aforementioned solutions, we propose a flexible runtime interleaver architecture to solve the memory conflict problem efficiently, so as to enable high throughput multi-standard turbo decoding.

\section{Eliminating Memory Reading Conflicts Via Balanced Turbo Decoding SCHEdULING}

In a traditional turbo decoding architecture, due to the existence of data dependencies during trellis traversal in a turbo decoder, SISO decoders should stall until the currently requested datum is ready. Frequent stalling of SISO decoders will significantly decrease the decoding throughput. In order to achieve high throughput, we should keep all the SISO decoders running at full speed without stalling. Fig. 5 demonstrates two cases showing traditionally how the memory conflict problem was solved using memory conflict solvers, when three SISO decoders are trying to access (write or read) the same memory module. We will analyze the disadvantage of these solutions, and then discuss our solution in Section $\amalg I I-B$.

\section{A. Properties of Memory Reading and Writing Conflicts}

For a memory writing conflict (Fig. 5a), a buffer structure can be used as a "write conflict solver" to cache the data accesses and resolve the memory conflicts. All the SISO decoders can execute at full speed so that the overall high throughput can be guaranteed. In contrast, if three SISO decoders request data from memory in the same clock cycle (Fig. 5b), only SISO decoder-1 can get the requested LLR value $L_{1}$ immediately. The SISO decoder- 2 must wait for a clock cycle to get $L_{2}$ and SISO decoder-3 must wait for two clock cycles to get $L_{3}$. Although buffering and data prefetching may help reduce the number of memory reading conflicts, it is difficult to completely eliminate memory reading conflicts in a practical hardware implementation. In addition, the reading conflict solver and the writing conflict solver are so different that implementing both of them utilizes more hardware resources and requires extra multiplexing logic. 


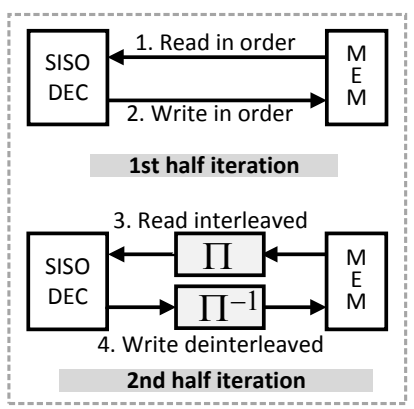

(a)

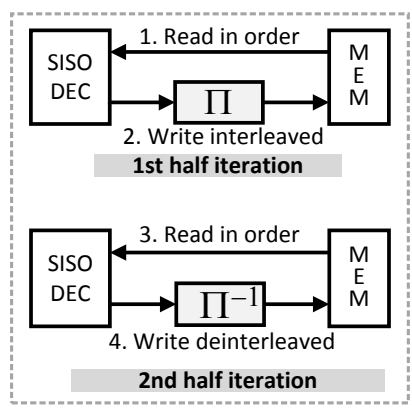

(b)

Fig. 6. (a) The unbalanced turbo decoder scheduling; (b) The balanced scheduling scheme. (The numbers indicate the order of scheduling steps.)

\section{B. Unbalanced and Balanced Scheduling Schemes}

In this Section, we describe the advantages of a balanced scheduling scheme to deal with the memory conflict problem. There are two scheduling schemes when implementing a turbo decoder, namely an unbalanced scheme and a balanced scheme. As shown in Fig. 6a, in an unbalanced scheduling scheme, the SISO decoder reads (writes) contiguous data from (to) the memory in a natural order in the 1st half iteration. During the 2nd half iteration, the SISO decoder first reads data from memory in an interleaved way; once computation is done, the SISO decoder writes the output data into memory in a deinterleaved way. Due to the randomness of the interleaving/deinterleaving algorithms, both memory reading and writing in the 2 nd half iteration suffer from memory contentions in a parallel turbo decoder.

Fig. $6 \mathrm{p}$ shows the balanced scheduling scheme in which SISO decoders write output data in the interleaved/deinterleaved order in both half iterations, so that in the next half iteration they can read the data in a naturally continuous order. Since all the memory reading operations are in-order, there is no memory conflict at all [1]. The balanced scheduling scheme can lead to more efficient designs than the unbalanced scheduling scheme because of the following reasons. First of all, the memory writing conflict is easier to solve than the memory reading conflict as is discussed in Fig. 5 . Secondly, without memory reading conflicts, we can remove the reading conflict solver hardware from the interleaver and reduce the hardware cost. Thirdly, the balanced scheduling method generates a more balanced workload between two half iterations. Since both the read and write conflict solvers introduce extra penalty clock cycles, the memory conflicts occurring in both memory reading and writing in a unbalanced scheduling scheme lead to more penalty clock cycles in the 2 nd half iteration. Furthermore, during the 2nd half iteration in unbalanced scheme, the control logic need to keep track of the execution status of SISO decoders, since the SISO decoders may run/stall independently. The bookkeeping and frequent stalling/restarting operations on the SISO decoders require more complex control logic in the unbalanced scheduling scheme. In the balanced scheduling scheme, the symmetric structure between two half iterations results in a more balanced workload, which simplifies the control logic.

Due to the above-mentioned benefits, we choose the bal-

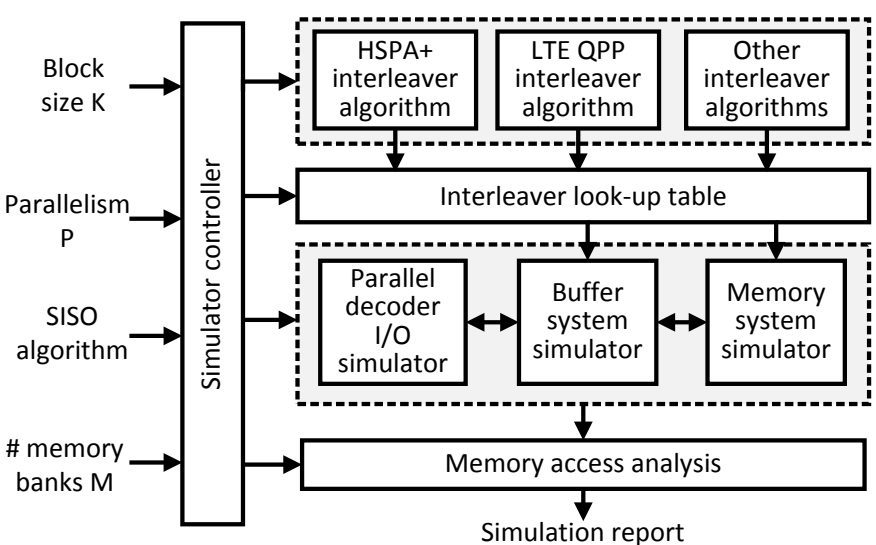

Fig. 7. Cycle-accurate simulation used to study the memory conflict problem.

anced scheduling scheme in our multi-standard turbo decoder implementation.

\section{Further Discussions}

Although the balanced scheduling scheme requires both interleaver and deinterleaver hardware modules, the fact that the interleaver and deinterleaver algorithms typically have symmetric structures implies that hardware sharing is possible to save computational resources and storage resources. Therefore, the overhead of a two-mode interleaver/deinterleaver would be small compared to a single-mode interleaver, if hardware sharing is explored. In addition, as an enabler for highly parallel turbo decoders supporting standards that do not employ contention-free interleaver algorithms (such as the HSPA+ standard), a small hardware overhead can be acceptable. This hardware sharing method will be further discussed in Section $\mathrm{V}$ and Section VI-A

\section{Double-buffer Contention-free ArChitecture FOR INTERCONNECTION NETWORK}

By using the balanced turbo decoding scheduling scheme, we eliminate the memory reading conflicts, but the memory writing conflicts still exist. In this section, we describe a double-buffer contention-free (DBCF) architecture to solve the memory writing conflict problem with low hardware complexity [16]. We first introduce the methodology and system model to analyze properties of memory conflicts and then describe the DBCF architecture in detail.

\section{A. Methodology and System Model}

To study the statistical property of memory conflicts under an interleaving law with a special parallel architecture, a cycleaccurate simulator is designed using a methodology shown in Fig. 7. The simulator is written in the $\mathrm{C}$ language, and can simulate the behaviors of SISO decoders' I/O ports, the buffer system and the memory system in each clock cycle. Interleaving address patterns are calculated and stored in a look-up table in the beginning of a simulation. The simulator can be easily extended to support new interleaving laws. This cycle-accurate simulator can also be used to verify or simulate a memory conflict solver by modifying the buffer system simulator. This 


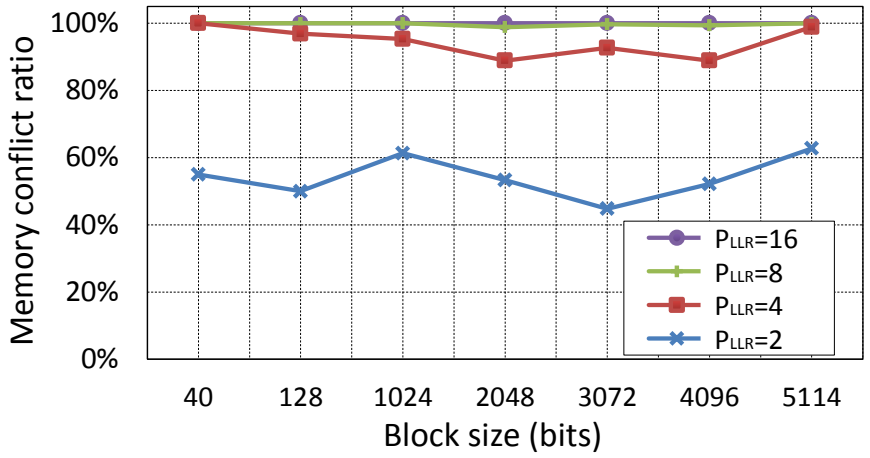

Fig. 8. Memory conflict ratio for different parallelism degrees and block sizes for the HSPA+ turbo code interleaver.

function is used to determine some architectural parameters of the DBCF architecture in Section IV-C.

Depending on the parallel decoding strategies and memory organizations, the memory access patterns can vary from case to case. Our implemented parallel turbo decoder consists of $P$ parallel Radix-4 XMAP decoders as described in Section II-B. and $M$ memory modules storing the extrinsic LLR values. In such a system, a Radix-4 XMAP decoder produces 4 new LLR data in each clock cycle. The total number of LLR data to be written in the extrinsic memory per clock cycle is defined as $P_{L L R}=4 P$, which can be considered as the effective parallelism of a parallel turbo decoder.

\section{B. Statistical Analysis of Memory Conflicts}

The HSPA+ mode is the most challenging part in a high throughput multi-standard turbo decoder due to the frequent and severe memory conflicts [20], [34]. Therefore, in order to design an efficient VLSI architecture, it is important to study the properties of memory conflicts in HSPA+ mode. We first analyze how the memory conflict ratio changes as the block sizes and the effective parallelism $P_{L L R}$ change by performing cycle-accurate simulations. Memory conflict ratio (MCR) is defined as a ratio of the number of clock cycles with memory conflicts to the total number of clock cycles for a half iteration: $M C R=N_{\text {conflict_cycle }} / N_{\text {total_cycle. The }}$ MCR can indicate how severe the memory conflict problem is for certain decoding configurations. Fig. 8 shows that for a fixed parallelism the MCR does not change significantly across different block size configurations. However, when parallelism $P_{L L R}$ goes higher, the MCR increases dramatically. For parallelism $P_{L L R}$ higher than 8 , memory conflicts occur in almost every clock cycle, which indicates very severe memory conflict problems. Therefore, as the parallelism goes higher, the difficulty to resolve the memory conflict problem increases drastically.

Simulation results show that during the whole decoding process, the average number of memory accesses for each memory module per clock cycle is very close to 1 . This implies the possibility to use buffers to smooth the bursty data requests to a memory module, and finally to achieve near $1 \mathrm{data} /$ cycle/memory throughput. We further analyze the statistical distribution of memory conflicts. If $n$ LLR data in

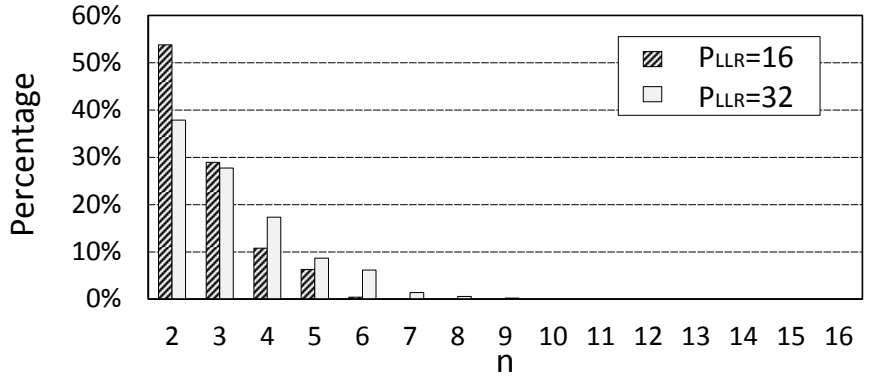

Fig. 9. The percentage of $n$-way memory conflicts (for $P_{L L R}=16$ and $P_{L L R}=32$ ). Interleaving algorithm from 3GPP HSPA+ standard is employed. Turbo code block size is $K=5114$.

the same memory module are accessed by multiple SISO decoders in one clock cycle, we call it an $n$-way memory conflict $\left(n \in\left[2, P_{L L R}\right]\right)$. The percentage of $n$-way memory conflicts

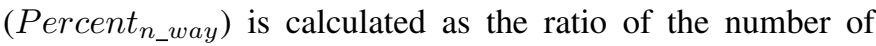
$n$-way conflicts $\left(N_{n \_w a y}\right)$ to the total number of memory conflicts $\left(N_{\text {Conflict }}\right)$ : Percent $n_{-}$way $=N_{n_{-} \text {way }} / N_{\text {Conflict }}$. The percentage of $n$-way memory conflicts for parallelism of 16 and 32 in 3GPP HSPA+ interleaver are shown in Fig. 9 We can notice that most of the memory conflicts are 2-, 3- and 4-way conflicts. These three types of conflicts cover $93.35 \%$ and $82.97 \%$ of the memory conflicts for $P_{L L R}=16$ and $P_{L L R}=32$, respectively. Based on the above observations, we propose a double-buffer contention-free architecture (DBCF) to efficiently solve the memory conflict problem.

\section{Double-buffer Contention-free (DBCF) Architecture}

The DBCF buffer architecture is built around the interleaver (with interleaving address generation units) between the SISO decoders and memory modules. Fig. 10 shows the diagram of the DBCF architecture. Major components of the DBCF architecture include FIFOs associated with SISO decoders, circular buffer associated with memory modules, buffer routers and bypass units. The name of DBCF architecture comes from the fact that two sets of buffers (FIFOs and circular buffers) are employed, which gives the proposed architecture a large advantage over the traditional single-buffer-based architectures [11], [27].

1) Micro-architecture of $D B C F$ : The main idea of the DBCF architecture is to use circular buffers to cache the concurrent data writing operations. The circular buffers should be implemented using registers in order to support concurrent data writing. A write pointer and a read pointer are provided to control the buffer access. If full inter-connection is fulfilled between the interleaver and the circular buffers, the exponentially growing hardware cost as the parallelism goes higher makes the implementation very inefficient [27]. According to the statistical analysis of memory conflicts, it is rare to have an $n$-way memory conflict for $n>4$, therefore, a selectionbased data routing scheme is implemented by the buffer router. We define a selection parameter $S\left(S \in\left[1, N_{L L R}\right]\right)$, which controls the number of data that are allowed to be routed to the circular buffers in a clock cycle. At any time, up to $S$ LLR data are allowed to access the circular buffer, the other 


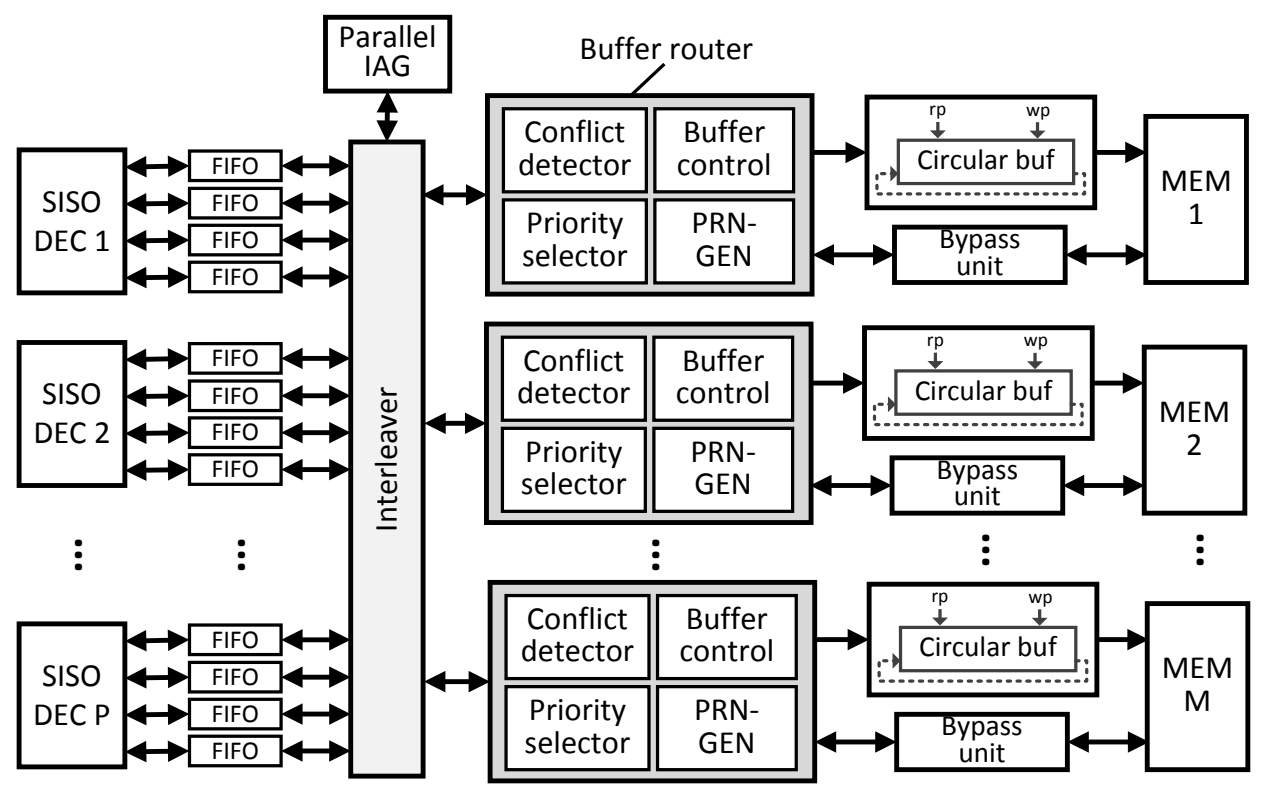

Fig. 10. Block diagram of the proposed DBCF buffer architecture to the solve memory writing conflict. (Note: wp and rp in the circular buffer represent write pointer and read pointer, respectively.)

$\max (0, n-S)$ LLR data are rejected for a $n$-way memory conflict case. For example, if there are 5 LLR data trying to access a certain memory module $(n=5)$. The conflict detector detects a memory conflict. Assume we have set $S=3,3$ out of 5 LLR data will be stored into the circular buffer associated with the memory module. At the same time, the remaining 2 LLR data $(\max (0, n-S)=\max (0,5-3)=2)$ are pushed into the FIFOs connected to the producer SISO decoders. This selection-based data routing method reduces complexity of the inter-connection network between the interleaver and the circular buffers from $P_{L L R} \times P_{L L R}$ to $P_{L L R} \times S$.

The conflict detector can detect the memory conflicts at runtime using a compare-select cluster. It is worth mentioning that in the proposed architecture memory conflicts are detected and solved at runtime, without any pre-stored data sets from the initial design phase. Then, with the help of pseudo-random number generator (PRN-GEN) circuits, priority selector circuits determine which $S$ data are selected with equal fairness. The buffer control units maintain the writing and reading operations to the circular buffers. The bypass units directly route the data into the memory module when the circular buffer is empty to avoid unnecessary latency caused by circular buffers.

The FIFOs associated with SISO decoders is one of the important reasons why the DBCF architecture can efficiently solve the memory writing conflict problem. If a traditional single-buffer architecture is used, we have to stall the corresponding SISO decoders if their LLR outputs are rejected by the buffer router circuits; otherwise, the rejected data will be lost. Thanks to the FIFOs introduced by the DBCF architecture, a rejected LLR datum is pushed into a FIFO associated with the SISO decoder that produced the rejected LLR datum.

2) Further Analysis of DBCF Architecture: The DBCF architecture can significantly reduce the memory usage for the

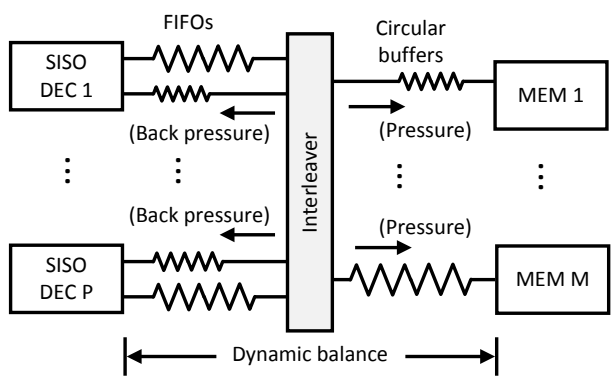

Fig. 11. Dynamic balance of data pressure on inter-connection network via "spring-like" structure of the DBCF architecture.

data buffers. A model in Fig. 11 can help further understand the principle behind the DBCF architecture. The two main components of the DBCF architecture, FIFOs and circular buffers, work collaboratively like two sets of springs which distribute data pressure evenly across both side of the interconnection network. Think about a possible case in which multiple SISO decoders send data concurrently to memory1 (MEM 1) in several consecutive clock cycles. This burst behavior generates high data pressure on the circular buffer associated with MEM 1. Therefore, if without FIFOs, a large circular buffer is required to handle the occasional burst data accesses, which leads to inefficient implementations. As a comparison, in DBCF architecture, we use small circular buffers. Even when the circular buffer is fully occupied by the bursty data, the incoming data are pushed into the FIFOs, so that the circular buffers will not overflow and we do not lose any data. That being said, the FIFOs and circular buffers together maintain a dynamic balance of the data pressure on the interconnection network. As a result, the size of the circular buffers can be significantly reduced. Moreover, since the rejected data are stored in several FIFOs associated with SISO decoders, with each FIFO accepting only one datum. 
TABLE I

SIMULATION RESULTS FOR PARAMETER DETERMINATION. HSPA+ INTERLEAVER, $K=5114, P_{L L R}=16$.

\begin{tabular}{c|c|c|c|c|c|c|c}
\hline \multicolumn{7}{c|}{ Simulation parameters } & \multicolumn{3}{|c}{ Results } \\
\hline$P_{L L R}$ & $M$ & $S$ & $D_{F I F O}$ & $D_{b u f}$ & $C_{0}$ & $C_{1}$ & $\Delta C$ \\
\hline \hline 16 & 16 & 1 & 0 & 128 & 320 & 495 & 175 \\
\hline \hline 16 & 16 & 3 & 4 & 12 & 320 & 332 & 12 \\
\hline 16 & 32 & 3 & 3 & 4 & 320 & 323 & 3 \\
\hline
\end{tabular}

TABLE II

SiMULATION RESUlTS FOR PARAMETER DETERMINATION. HSPA+ INTERLEAVER, $K=5114, P_{L L R}=32$.

\begin{tabular}{c|c|c|c|c|c|c|c}
\hline \multicolumn{6}{|c|}{ Simulation parameters } & \multicolumn{3}{c}{ Results } \\
\hline$P_{L L R}$ & $M$ & $S$ & $D_{F I F O}$ & $D_{b u f}$ & $C_{0}$ & $C_{1}$ & $\Delta C$ \\
\hline \hline 32 & 32 & 1 & 0 & 128 & 160 & 268 & 108 \\
\hline \hline 32 & 32 & 3 & 8 & 12 & 160 & 170 & 10 \\
\hline 32 & 64 & 3 & 4 & 7 & 160 & 164 & 4 \\
\hline
\end{tabular}

The data back pressure is gently distributed across FIFOs, so that small FIFO size is sufficient.

In addition to the function of pressure balancing, the insertion of FIFOs also decouples the SISO decoders from the interleaver. This is important because of the following reasons: (1) with DBCF architecture, SISO decoders can run at full speed without stalling, no matter how severe the memory conflicts occur on the right hand side of interleaver. This is a key to achieving high throughput; (2) any SISO decoder architecture is supported to achieve high throughput. For instance, a Radix-4 XMAP decoder generates 4 LLR data per clock cycle, among which some may be successfully written into a memory module, while others may be pushed back to FIFOs. But the Radix-4 XMAP decoder keeps producing new outputs all the time. Without the help of the proposed doublebuffer architecture, a Radix-4 XMAP decoder has to stall even if only 1 LLR value (out of 4 ) is rejected by a buffer router; (3) The decoupling between SISO decoders and interleaver also reduces the complexity of the SISO decoders since no feedback control from interleaver (or memory modules) to the SISO decoders is needed.

3) Determining Architectural Parameters: To determine a parameter configuration (selection parameter $S$, FIFO depth $D_{F I F O}$, circular buffer depth $D_{b u f}$ ) for a parallel turbo decoder with parallelism of $P_{L L R}$ and $M$ memory modules, the cycle-accurate simulation is performed by adding the DBCF architecture to the buffer system simulator. By changing different combinations of $\left(S, D_{F I F O}, D_{b u f}\right)$, we can tune the design to meet different performance/complexity goals in terms of memory usage, decoding latency, and complexity of the interconnection network. In Table [ and Table II parameter configurations for $P_{L L R}=16$ and $P_{L L R}=32$ to achieve high throughput goal as well as the corresponding simulation results are shown. Here, we show results for the largest block size in HSPA+ standard $(K=5114)$, which typically causes more serious memory conflict problem and longer latency. In these tables, $M$ denotes number of banks in the extrinsic memories; $C_{0}$ denotes the number of clock cycles to decode a codeword in an ideal case without any memory conflict; $C_{1}$ denotes the actually clock cycles including the clock cycles used to solve memory conflicts; $\Delta C$ denotes extra clock cycles actually used compared to the ideal case $\left(\Delta C=C_{1}-C_{0}\right)$. Although all SISO decoders run at full speed without stalling in the proposed architecture, the FIFOs and circular buffers still need a few clock cycles to unload data, which causes extra clock cycles $\Delta C$. A well-tuned parameter configuration can lead to small $\Delta C$. Taking Table [I] as an example, the first row provides a baseline case in which only a large circular buffer (without priority selection and FIFOs) is used to cache the bursty data. In this baseline case, with buffer size set to $128\left(D_{b u f}=128\right), 108$ extra clock cycles are needed to finish decoding. The second row shows the usage of priority selection with $S=3, D_{F I F O}=8$ and $D_{b u f}=12$. In this case, 10 extra cycles are needed. The third row shows the case in which sub-banking techniques (here, we set $M=2 \times P_{L L R}$ for sub-banking) are used to further reduce $D_{F I F O}$ (reduced to 4) and $D_{b u f}$ (reduced to 7), and only 4 extra clock cycles are required to finish the decoding. Table I and Table II have also demonstrated the effectiveness and efficiency of the proposed DBCF architecture in terms of the reduced buffer sizes and decreased extra clock cycles.

\section{EFFICIENT Unified INTERLEAVER/DEINTERLEAVER ARCHITECTURE}

To improve the flexibility and configurability of a turbo decoder, it is of great interest but also quite challenging to design and implement an on-the-fly interleaving address generator (IAG) supporting both interleaving and deinterleaving modes for multiple standards. In this section, we propose a unified parallel IAG architecture to support both interleaver and deinterleaver algorithms with low hardware overhead.

We notice that many interleaving laws in standards have similar computation kernels for both interleaving and deinterleaving. For instance, a pseudo-random column-row permutation algorithm is employed in 3GPP UMTS/HSPA+ standards [2]. The core operation of the HSPA+ interleaver can be summarized as computing a column index function $U_{i}(j)$ from the original column $j$ after intra-row permutation $j \rightarrow U_{i}(j)$ for the $i$-th row, using the formula $U_{i}(j)=s\left(\left(j \times r_{i}\right)\right.$ $\bmod (P-1))$. Similarly, it has been proved that the core operation in the UMTS/HSPA+ deinterleaver is to compute an intra-row permutation $j^{\prime} \rightarrow U_{i}^{-1}\left(j^{\prime}\right)$ using formula $U_{i}^{-1}\left(j^{\prime}\right)=$ $\left(s^{-1}\left(j^{\prime}\right) \times m_{i}\right) \bmod (P-1)$ [40]. The core computation units in the interleaver and deinterleaver can be summarized as the same computation kernel $(a \times b) \bmod c$. Furthermore, most of the intermediate values precomputed by the pre-processing units can be reused, except for only a few intermediate values used exclusively by the deinterleaver mode.

Similar observations can be made by investigating the LTE QPP (Quadratic Permutation Polynomial) interleaving algorithm [3]. The QPP interleaver implements a permutation based on the quadratic polynomial $f(x)=\left(f_{1} x+f_{2} x^{2}\right)$ mod $K$. Efficient implementations by recursively computing $f(x)$ have been proposed [17], [19]. Researchers have proved that the inverse permutation in the form of quadratic polynomials exist for most of the $K$ (153 out of 188), and for other $K$ inverse permutation in the forms of cubic (31/188) or quartic (4/188) polynomials can be found [41], [42]. Similarly, 


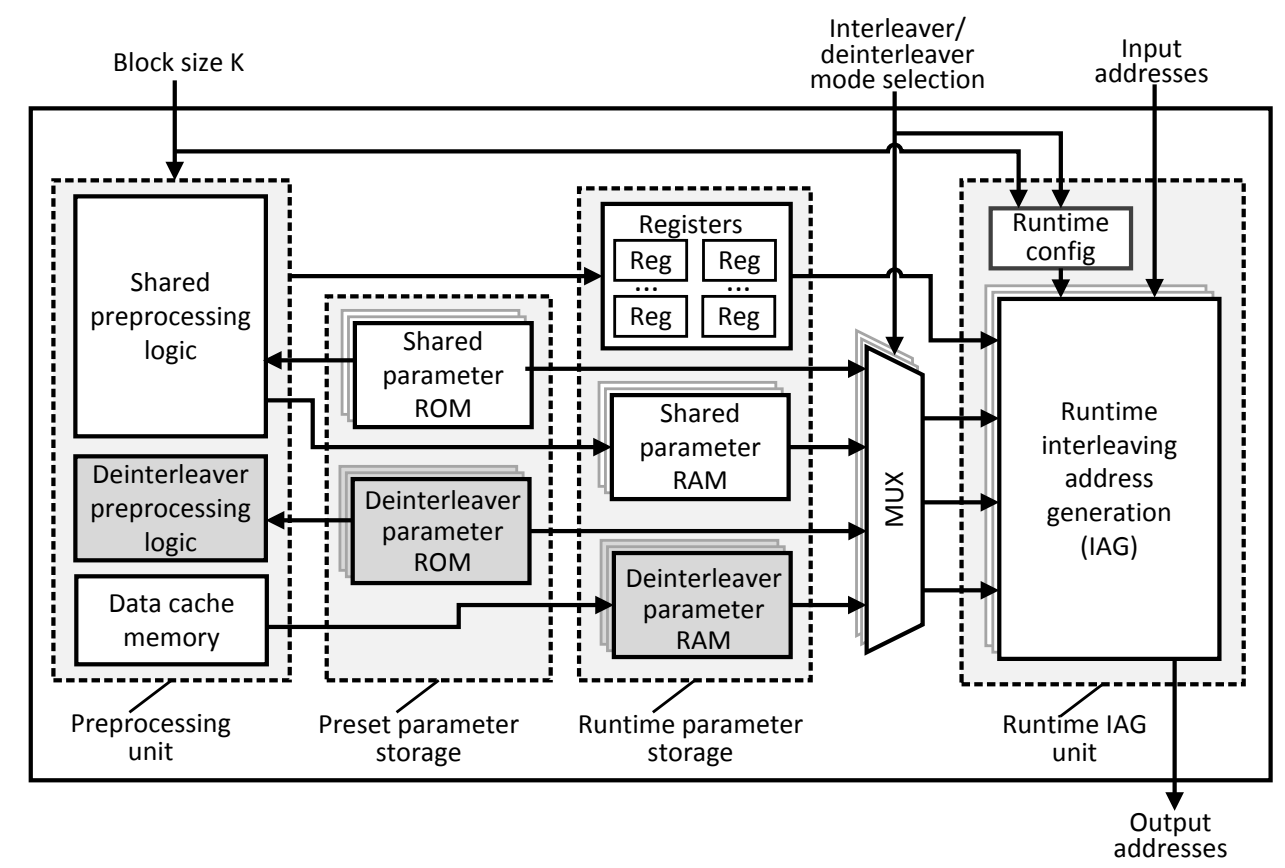

Fig. 12. Architecture of unified parallel interleaving address generation (IAG) supporting both interleaving and deinterleaving. Note that we need $P_{L L R}$ copies of each duplicated block in this figure.

the deinterleaving addresses can also be computed recursively using efficient hardware. Therefore, the QPP interleaver and deinterleaver can also share the kernel computation unit $((a+b x) \bmod c)$.

Based on the above observations, we propose a unified interleaver/deinterleaver architecture which shares a large portion of the hardware between the interleaver and deinterleaver modes, leading to only a small overhead compared to a single-mode interleaver. Fig. 12 shows the proposed unified parallel IAG architecture, which consists of the following key blocks: (1) preset parameter storage, (2) preprocessing unit, (3) runtime parameter storage, and (4) runtime IAG block. Here, let us take the column-row random interleaving algorithm in the HSPA+ standard as an example to explain how these blocks work. The algorithm details and notation definitions can be found from 3GPP UMTS/HSPA+ standards [2] or references [22], [40]. The preset parameter storage implemented using ROMs contains static parameters such as interrow permutation pattern $T_{i}(128 \times 5 b i t \mathrm{tROM})$ and permutated sequence $r_{i}(1440 \times 7 b i t$ rROM $)$ used by both modes, and the modular multiplicative inverse sequence $m_{i}(1200 \times 7 b i t$ $\mathrm{mROM}$ ) used exclusively by the deinterleaver [40]. The preprocessing unit computes the parameters used in the runtime IAG block. The preprocessing unit first generates several runtime parameters based on the block size $K$, such as the number of rows/columns $(R / C)$ of the permutation matrix, and then stores them in registers. It also produces permutation patterns such as the base sequence for intra-row permutations $s_{i}(256 \times 8 b i t$ SRAM $)$ and the corresponding inverse base sequence used in the deinterleaver mode $s_{i n v, i}(256 \times 8 b i t$ SRAM). Finally, the runtime IAG block, which consists of computation and control logic, converts input addresses to the interleaved/deinterleaved addresses. Based on the input block size $K$ and interleaver/deinterleaver mode selection, the runtime IAG can be configured to work in the corresponding mode.

As is shown in Fig. 12, we need to duplicate some memory and computation units for $P_{L L R}$ times to generate $P_{L L R}$ parallel addresses in one clock cycle. It is worth noting that we only need one single copy of the preprocessing hardware. The balanced scheduling scheme proposed in Section III makes it possible that the parallel interleaver/deinterleaver can function in a time-division multiplexing manner (different half iterations, to be specific). Therefore, a significant amount of hardware resources for two modes can be shared between two half iterations, which include the preprocessing unit, kernel computation units inside the runtime IAG, as well as ROMs and RAMs storing necessary parameter arrays. The hardware sharing feature has been integrated in our turbo decoder implementation presented in Section VI, which can demonstrate the efficiency of the proposed unified IAG architecture.

\section{VLSI IMPLEMENTATION}

To show the effectiveness of the proposed architecture, we implement a high throughput HSPA+/LTE multi-standard turbo decoder as is shown in Fig. 13. The design goal is to achieve more than $1 \mathrm{Gbps}$ throughput for LTE mode and 672 Mbps throughput for HSPA+ mode.

The Radix-4 XMAP decoding architecture described in Section II-B is used to implement the SISO decoders. The max$\log$-MAP algorithm is employed with a scaling factor 0.75 applied to improve the decoding performance [43]. In the SISO decoder, fixed-point representation is used for state metrics and LLR data as follows: 5-bit channel LLR values, 6-bit extrinsic LLR values; 9-bit branch metrics $(\gamma)$, and 10-bit state metrics $(\alpha$ and $\beta$ ). Fig. 14 shows the fixed-point simulation results for both HSPA+ and LTE turbo decoder using the proposed 


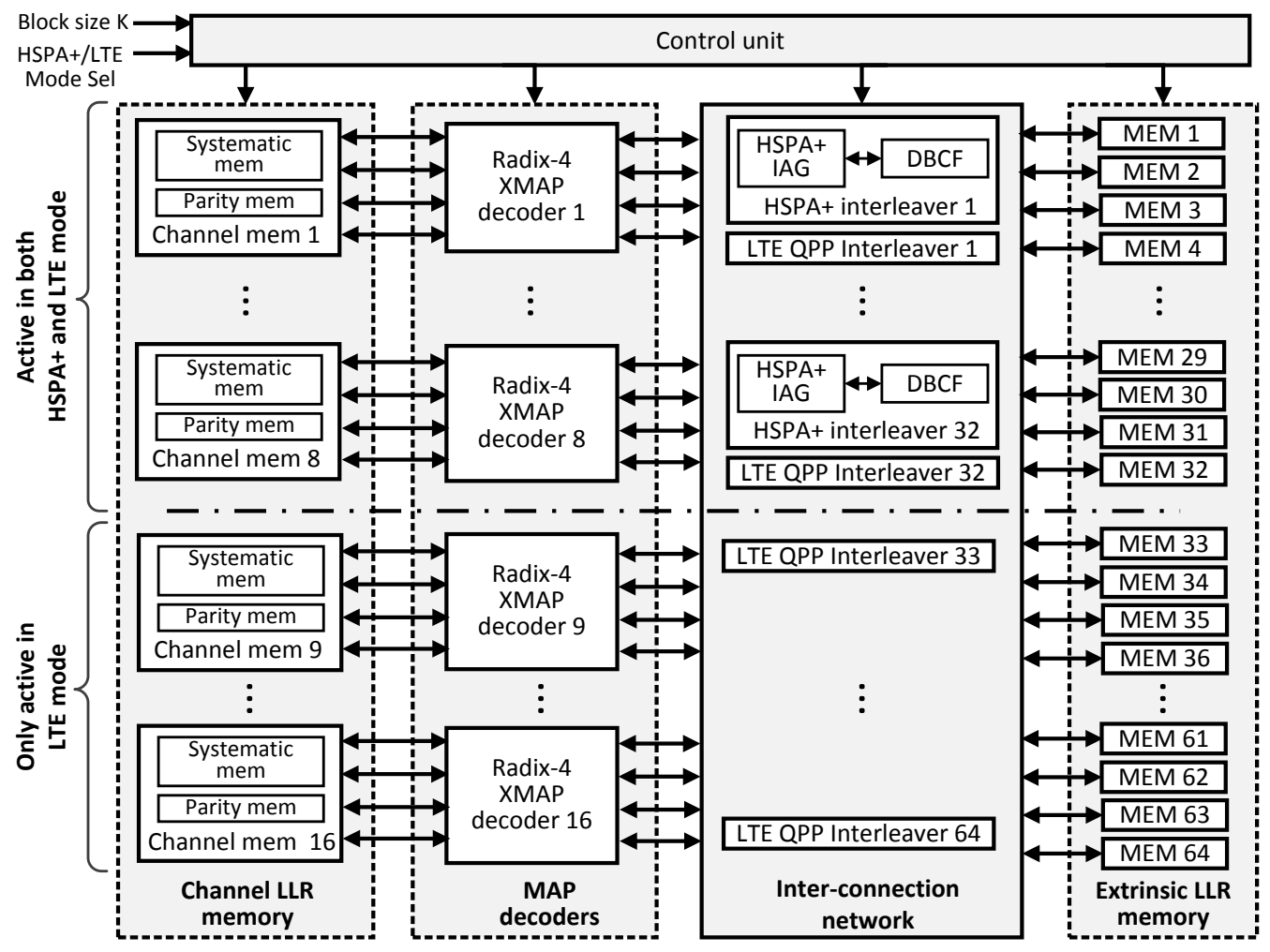

Fig. 13. Overall architecture of the proposed multi-standard (HSPA+/LTE/LTE-Advanced) turbo decoder.

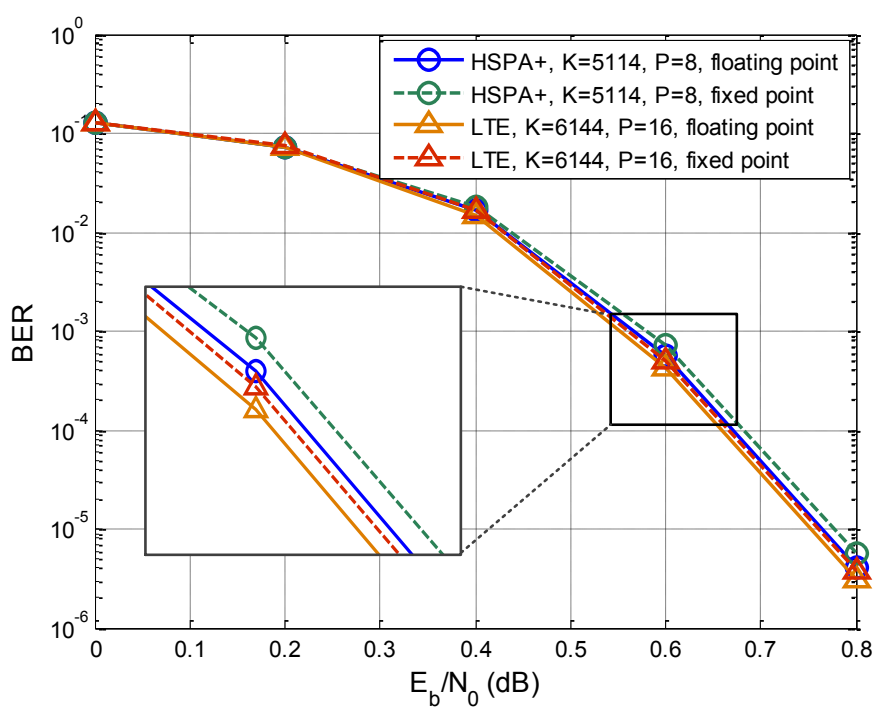

Fig. 14. Bit error rate (BER) performance with fixed-point simulation for turbo decoder (code rate 1/3) with the proposed parallel architecture, using BPSK modulation over an additive white Gaussian noise (AWGN) channel. The parallelism degrees for HSPA+ and LTE mode are 8 and 16, respectively. The number of iterations is 5.5 .

architecture and quantization. The simulation results show that the fixed-point performance degradation is less than $0.1 \mathrm{~dB}$.

To achieve the throughput goal, the turbo decoder consists of $P=16$ Radix-4 XMAP decoders, which results in an effective parallelism of $64\left(P_{L L R}=P \times 4=64\right)$. Thus, the extrinsic LLR memory is partitioned into 64 modules. Each extrinsic LLR memory module is implemented using a
$\left(K / P_{L L R} \times B_{\text {ext }}\right)$ two-port SRAM ( $B_{\text {ext }}$ is the word length of the extrinsic LLR). Moreover, 16 channel LLR memory modules are used to store the input channel LLR values. Each channel LLR memory module is a $\left(3 K / P \times 2 B_{c h}\right)$ singleport SRAM ( $B_{c h}$ is the word length of the channel LLR). Every channel LLR memory module is further partitioned into several banks to provide parallel accesses to the systematic and parity check values. To support the high throughput of LTE mode, all 16 SISO decoders work actively during decoding, with the corresponding 64 extrinsic LLR memory modules, 16 channel LLR memory modules, and 64 QPP interleavers. The HSPA+ interleaver and DBCF buffer architecture will be bypassed under LTE mode. On the other hand, when configured to work under the HSPA+ mode, the turbo decoder uses 8 SISO decoders leading to an effective parallelism of $P_{L L R}=32$. Accordingly, 32 extrinsic LLR memory modules, 8 channel LLR memory modules, and 32 HSPA+ interleavers are employed. One DBCF buffer structure per extrinsic LLR memory module is implemented to handle the memory conflicts. The remaining 8 SISO decoders along with memory modules are disabled using HSPA+ mode.

The multi-standard HSPA+/LTE turbo decoder, which is described using Verilog HDL, has been synthesized, placed and routed with the TSMC 45nm CMOS technology. In this section, we will describe the implementation results in detail and comparisons with related work. 
TABLE III

COMPARISON WITH EXISTING UMTS/HSPA+ INTERLEAVERS. (NOTE: THE CHIP AREA INCLUDES IAG AND INTERCONNECTION-NETWORK, EXCEPT FOR THE FIRST THREE PUBLICATIONS.)

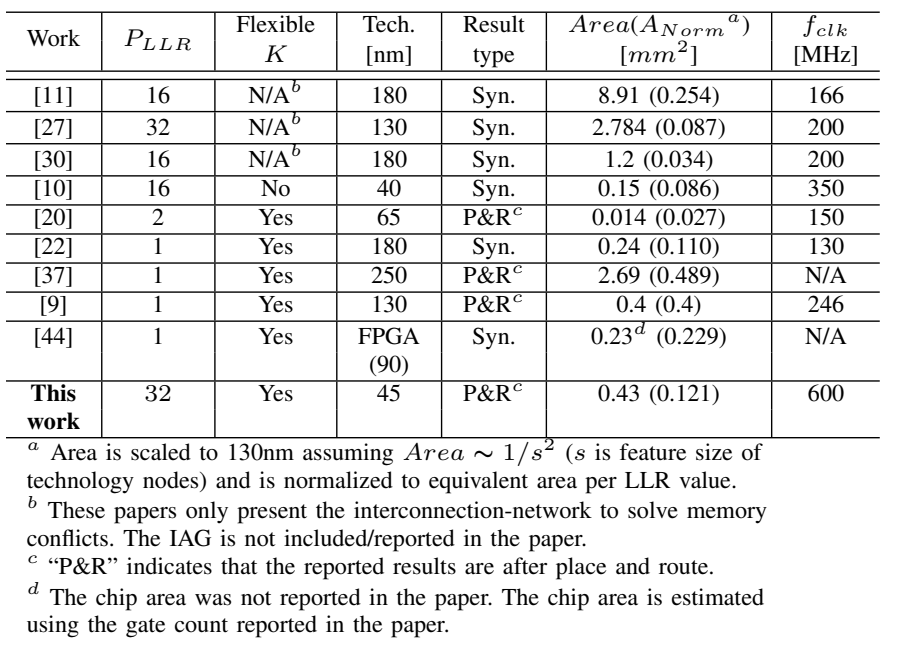

\section{A. Implementation Results for the Contention-free HSPA+ Interleaver}

The HSPA+ interleaver is the most critical part in the proposed multi-standard turbo decoder design. To achieve up to $672 \mathrm{Mbps}$ data rate under HSPA+ mode, the turbo decoder employs an effective parallelism of $P_{L L R}=32$, which can result in severe memory conflicts as indicated by Fig. 8 Therefore, we first present implementation results for the HSPA+ interleaver.

The HSPA+ interleaver is implemented based on the balanced scheduling scheme, the DBCF buffer architecture and the unified parallel interleaver/deinterleaver architecture described in the previous sections. The block size $K$ can be configured at runtime. When a new block size $K$ is set, the preprocessing unit re-calculates the runtime parameters. The interleaver can work at both interleaver and deinterleaver modes based on a mode selection signal.

As a reference design, we first implemented a singlefunction IAG which only supports the interleaving algorithm. The chip area is $0.249 \mathrm{~mm}^{2}$ when synthesized with the TSMC $45 \mathrm{~nm}$ CMOS technology and targeting $600 \mathrm{MHz}$ clock frequency. Then we implemented a dual-function IAG supporting both the interleaving and deinterleaving algorithms of the HSPA+ standard. The area for the unified IAG is $0.278 \mathrm{~mm}^{2}$. Thus, compared to the single-function IAG, the chip area of the dual-function IAG is only increased by $0.029 \mathrm{~mm}^{2}$, which indicates a $11.6 \%$ complexity overhead compared to a singlefunction IAG. This result has demonstrated that the proposed unified parallel IAG architecture enables great hardware sharing, leading to an efficient hardware implementation. Inside the unified parallel IAG, the preprocessing logic utilizes $806 \mu \mathrm{m}^{2}$ chip area, and the runtime computing logic utilizes $0.097 \mathrm{~mm}^{2}$ to generate 32 parallel interleaved (or deinterleaved) addresses in one clock cycle. The unified HSPA+ IAG uses $65 \mathrm{~Kb}$ memory as runtime parameter storage to support parallelism degree of 32 and different block sizes.

Table III summarizes the key features of the implemented

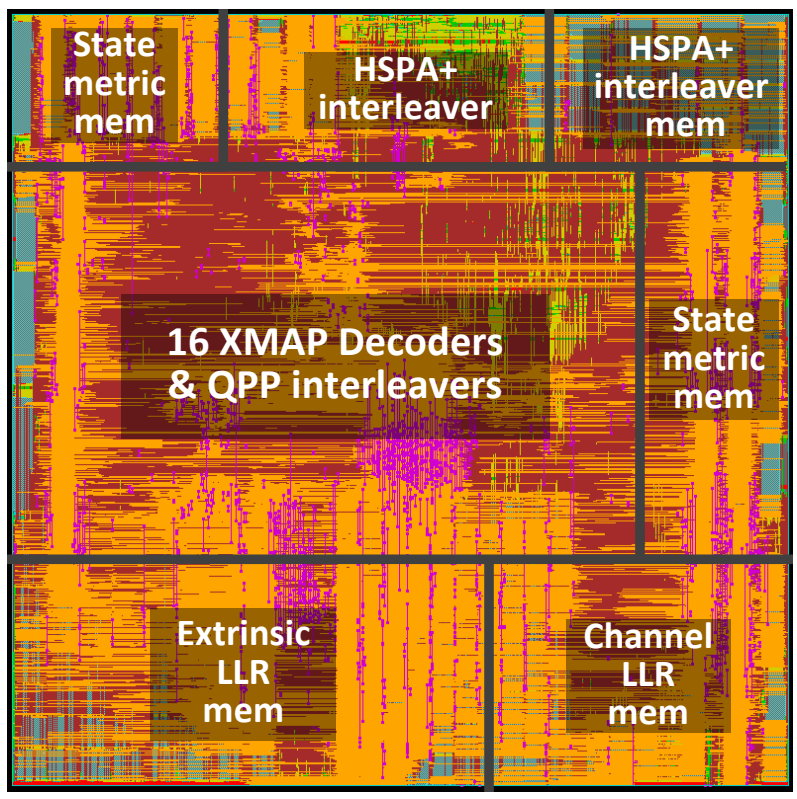

Fig. 15. VLSI chip layout for an HSPA+/LTE multi-standard turbo decoder with the architecture shown in Fig. 13 The TSMC 45nm standard cell library is used. This turbo decoder contains 16 Radix-4 XMAP decoders, 64 LTE QPP interleavers, and 32 HSPA+ interleavers.

contention-free HSPA+ interleaver, as well as the comparison with related work. All the work shown in Table IIII focus on the design of high performance and efficient interleavers for the 3GPP UMTS/HSPA+ turbo decoder. Among them, references [10], [11], [20], [27], [30] concentrate on the interconnection-network solving the memory conflict problem, so they did not report the area for the IAG modules. While the others present efficient solutions for IAG modules for the UMTS/HSPA+. To make a fair comparison, we show the normalized chip area for each implementation; furthermore, for those supporting parallel interleaving address generation, the chip area is further normalized by the supported parallelism to determine an equivalent chip area for each generated interleaving address. The results in [9], [22], [37], [44] support on-the-fly interleaving address generation for HSPA+, but they do not support parallel decoding due to the lack of a memory conflict solver. The interleavers in [10], [11] are not reconfigurable because they support only one block size. Only [10], [44] and our work support both the interleaver and deinterleaver modes. The architecture in [20] can support a parallel turbo decoder with different block sizes, but the architecture is limited to turbo decoders with low parallelism. Our proposed architecture shows the best tradeoff among flexibility, configurability, and hardware complexity.

\section{B. Implementation Results of Multi-Standard Turbo Decoder}

The key characteristics of the implemented multi-standard turbo decoder are summarized in Table IV] The design is synthesized using the TSMC $45 \mathrm{~nm}$ standard cell library. The chip core area of the implemented turbo decoder is $2.43 \mathrm{~mm}^{2}$. A top-level chip layout view after place and route is shown in Fig. 15. The maximum number of iterations of this turbo decoder is set to 5.5 . The block size $K$ can be configured 
TABLE IV

VLSI IMPLEMENTATION COMPARISON WITH EXISTING HIGH SPEED UMTS/HSPA+ AND MULTI-MODE TURBO DECODERS.

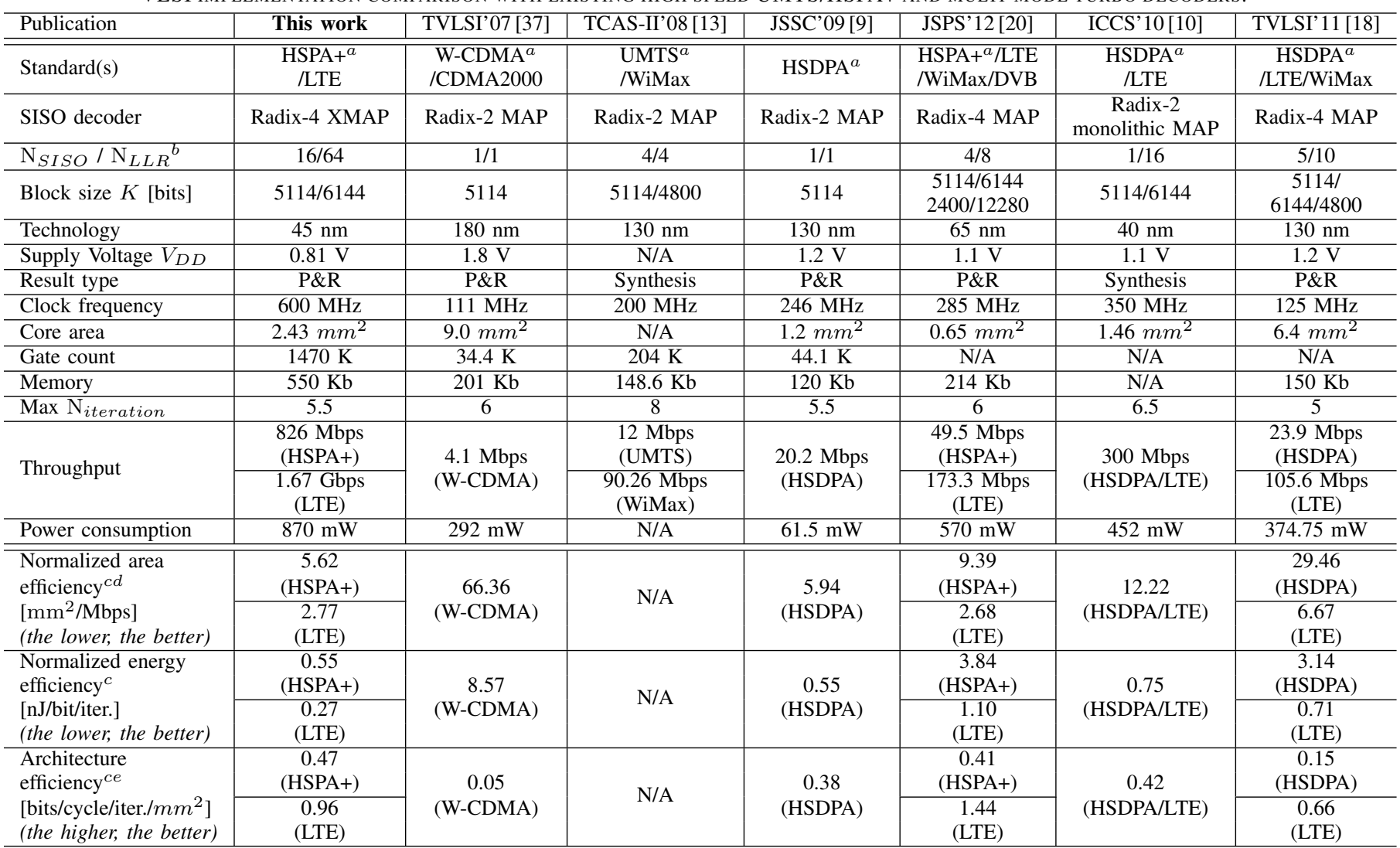

${ }^{a}$ The W-CDMA, HSDPA and HSPA+ are terms referring to different generations of technologies used in the 3GPP UMTS standard.

They all use the same turbo code structure and interleaving algorithms.

${ }^{b} N_{L L R}$ denotes the number of parallel LLR values produced per clock cycle, which represents the effective parallelism of a turbo decoder.

$c$ Technology is scaled to $130 \mathrm{~nm}$ CMOS assuming: Area $\sim 1 / s^{2}$, and $t_{p d}$ (propagation delay) $\sim 1 / s$. ( $s$ : feature size of a technology node).

Throughput is linearly scaled to 5.5 iterations.

${ }^{d}$ Normalized area efficiency $=$ Area $_{\text {Norm }} /$ Throughput $_{\text {Norm }}[17]-[19],[45]$.

${ }^{e}$ Architecture efficiency $=$ Throughput $\times N_{\text {iter }} /$ Area $_{N o r m} / f_{\text {clk }}^{[23]}$.

at runtime $(K=40 \sim 5114$ for HSPA+; $K=40 \sim 6144$ for LTE). When clocked at $600 \mathrm{MHz}$, a maximum throughput of $1.67 \mathrm{Gbps}$ can be achieved under the LTE mode with all 16 Radix-4 XMAP decoders running. While in the HSPA+ mode, a maximum throughput of $826 \mathrm{Mbps}$ can be achieved with 8 Radix-4 XMAP decoders active. Compared to a singlemode LTE turbo decoder, the area overhead of the HSPA+/LTE multi-standard turbo decoder is approximately $17 \%$ in our implementation $\left(0.426 \mathrm{~mm}^{2} / 2.43 \mathrm{~mm}^{2}\right)$.

To the best of our knowledge, our multi-standard turbo decoder implementation is the first to support the peak data rate of $336 \mathrm{Mbps}$ in Release 11 of the 3GPP UMTS/HSPA+ standard [2]. Moreover, our implementation also exceeds the $672 \mathrm{Mbps}$ peak data rate requirement of the potential future extension of the HSPA+ standard and the $1 \mathrm{Gbps}$ peak data rate requirement for the LTE-Advanced standard [3].

A comparison with related work can be found in Table IV] These related publications are either high speed UMTS/HSPA+ turbo decoder designs or multi-standard turbo decoders supporting the UMTS/HSPA+ standard. Implementation details such as clock frequency, chip core area, throughput, and power consumption are included. We adopt three normalized metrics to evaluate implementation efficiency: area efficiency, energy efficiency and architecture efficiency [17][19], [23], [45]. The normalization is done by scaling the reported throughput and area numbers to $130 \mathrm{~nm}$ CMOS technology. From Table IV, we can see that our implementation shows the best normalized energy efficiency among the publications under both HSPA+ and LTE mode. In HSPA+ mode, our implementation shows the best area (or architecture) efficiency. While for LTE mode, our implementation has better normalized area (or architecture) efficiency than most of papers listed in Table [V] Note that only [20] shows better area (or architecture) efficiency. However, it has lower energy efficiency than the proposed implementation. Moreover, the parallel interleaver architecture proposed by [20] only supports low parallelism for the HSPA+ mode, which limits its achievable throughput under HSPA+ mode.

\section{CONCLUSION}

In this paper, we propose a VLSI architecture for highly parallel turbo decoding aiming at multi-standard 3G/4G wireless communication systems. We first utilize the balanced scheduling scheme to avoid memory reading conflicts. Then, based on the statistical property of memory conflicts, we 
propose a double-buffer contention-free (DBCF) buffer architecture to eliminate memory writing conflicts. Furthermore, we propose an efficient unified parallel interleaver architecture supporting both interleaving and deinterleaving modes. It is worth mentioning that the proposed parallel interleaver architecture is independent from the SISO decoder architectures, the interleaver algorithms, and parallelism degrees, thus, providing good flexibility and scalability. To demonstrate the effectiveness of our design approaches, we synthesize, place and route an ASIC design for the HSPA+/LTE/LTE-Advanced multistandard turbo decoder using the proposed VLSI architecture with a $45 \mathrm{~nm}$ CMOS technology. The chip core area is a $2.43 \mathrm{~mm}^{2}$. When clocked at $600 \mathrm{MHz}$, this turbo decoder can achieve a maximum throughput of $826 \mathrm{Mbps}$ in HSPA+ mode, and a peak throughput of $1.67 \mathrm{Gbps}$ in LTE/LTE-Advanced mode. The implementation results show that as an enabler, the proposed parallel interleaver architecture is able to lead to highly parallel but also hardware-efficient turbo decoding implementations.

\section{REFERENCES}

[1] G. Wang, A. Vosoughi, H. Shen, J. R. Cavallaro, and Y. Guo, "Parallel interleaver architecture with new scheduling scheme for high throughput configurable turbo decoder," in Proc. IEEE Int. Symp. Circuits and Syst. (ISCAS), May 2013, pp. 1340-1343.

[2] The 3rd Generation Partnership Project (3GPP), "Technical specification group radio access network; multiplexing and channel coding (FDD), Tech. Spec. 25.212 Release-11,” 2012.

[3] _ , "Evolved universal terrestrial radio access (E-UTRA); multiplexing and channel coding, Tech. Spec. 36.212 Release-11," 2012.

[4] Qualcomm Incorporated, "HSPA+ Advanced taking HSPA+ to the next level," 2012. [Online]. Available: http://www.qualcomm.com/media/ documents/hspa-advanced-taking-hspa-next-level-0

[5] M. Maternia, M. Januszewski, K. Ranta-Aho, J. Wigard, A. Bohdanowicz, M. Marzynski, and M. Wcislo, "On performance of long term HSPA evolution: Towards meeting IMT-Advanced requirements," in Proc. IEEE Int. Conf. Commun. (ICC), Jun. 2012, pp. 6040-6044.

[6] C. Berrou, A. Glavieux, and P. Thitimajshima, "Near Shannon limit error-correcting coding and decoding: Turbo-codes." in Proc. IEEE Int. Conf. Commun. (ICC), May 1993, pp. 1064-1070.

[7] Z. Wang, Z. Chi, and K. K. Parhi, "Area-efficient high-speed decoding schemes for turbo decoders," IEEE Trans. VLSI Syst., vol. 10, no. 6, pp. 902-912, 2002.

[8] B. Bougard, A. Giulietti, V. Derudder, J.-W. Weijers, S. Dupont, L. Hollevoet, F. Catthoor, L. Van der Perre, H. De Man, and R. Lauwereins, "A scalable $8.7 \mathrm{~nJ} / \mathrm{bit} 75.6 \mathrm{Mb} / \mathrm{s}$ parallel concatenated convolutional (turbo-) codec," in Proc. IEEE Int. Conf. Solid-State Circuits (ISSCC), 2003, pp. 152-484.

[9] C. Benkeser, A. Burg, T. Cupaiuolo, and Q. Huang, "Design and optimization of an HSDPA turbo decoder ASIC," IEEE J. Solid-State Circuits, vol. 44, no. 1, pp. 98-106, Jan. 2009.

[10] T. Ilnseher, M. May, and N. Wehn, "A multi-mode 3GPP-LTE/HSDPA turbo decoder," in Proc. IEEE Int. Conf. Commun. Syst. (ICCS), Nov. 2010, pp. 336-340

[11] M. J. Thul, F. Gilbert, T. Vogt, G. Kreiselmaier, and N. Wehn, "A scalable system architecture for high-throughput turbo-decoders," $J$. VLSI Signal Process., vol. 39, pp. 63-77, 2005.

[12] P. Salmela, R. Gu, S. S. Bhattacharyya, and J. Takala, "Efficient parallel memory organization for turbo decoders," in Proc. European Signal Process. Conf., Sept. 2007, pp. 831-835.

[13] M. Martina, M. Nicola, and G. Masera, "A flexible UMTS-WiMax turbo decoder architecture," IEEE Trans. Circuits and Syst. II: Express Briefs, vol. 55, no. 4, pp. 369-373, 2008.

[14] M. May, T. Ilnseher, N. Wehn, and W. Raab, "A 150Mbit/s 3GPP LTE turbo code decoder," in Des., Autom. \& Test in Europe (DATE), 2010, pp. $1420-1425$.
[15] C.-C. Wong, M.-W. Lai, C.-C. Lin, H.-C. Chang, and C.-Y. Lee, "Turbo decoder using contention-free interleaver and parallel architecture," IEEE J. Solid-State Circuits, vol. 45, no. 2, pp. 422-432, 2010.

[16] G. Wang, Y. Sun, J. R. Cavallaro, and Y. Guo, "Concurrent interleaver architecture for high throughput multi-standard parallel turbo decoder," in Proc. IEEE Int. Conf. Application-Specific Systems, Architectures and Processors (ASAP), Sept. 2011, pp. 113-121.

[17] C. Studer, C. Benkeser, S. Belfanti, and Q. Huang, "Design and implementation of a parallel turbo-decoder ASIC for 3GPP-LTE," IEEE J. Solid-State Circuits, vol. 46, no. 1, pp. 8-17, 2011.

[18] C.-H. Lin, C.-Y. Chen, and A.-Y. Wu, "Area-efficient scalable MAP processor design for high-throughput multistandard convolutional turbo decoding," IEEE Trans. VLSI Syst., vol. 19, no. 2, pp. 305-318, 2011.

[19] Y. Sun and J. R. Cavallaro, "Efficient hardware implementation of a highly-parallel 3GPP LTE/LTE-advance turbo decoder," VLSI J. Integration, vol. 44, no. 4, pp. 305-315, 2011.

[20] R. Asghar, D. Wu, A. Saeed, Y. Huang, and D. Liu, "Implementation of a Radix-4, parallel turbo decoder and enabling the multi-standard support," Springer J. Signal Process. Syst., vol. 66, no. 1, pp. 25-41, 2012.

[21] A. Sani, P. Coussy, and C. Chavet, "A first step toward on-chip memory mapping for parallel turbo and LDPC decoders: A polynomial time mapping algorithm," IEEE Trans. on Signal Process., vol. 61, no. 16, pp. 4127-4140, 2013

[22] Z. Wang and Q. Li, "Very low-complexity hardware interleaver for turbo decoding," IEEE Trans. Circuits Syst. II, vol. 54, no. 7, pp. 636-640, Jul. 2007.

[23] P. Murugappa, A. Baghdadi, and M. Jézéquel, "Parameterized areaefficient multi-standard turbo decoder," in Des., Autom. \& Test in Europe (DATE), Mar. 2013, pp. 109-114.

[24] S. Belfanti, C. Roth, M. Gautschi, C. Benkeser, and Q. Huang, "A 1Gbps LTE-advanced turbo-decoder ASIC in 65nm CMOS," in Symp. on VLSI Circuits (VLSIC), June 2013, pp. C284-C285.

[25] A. Giulietti, L. van der Perre, and A. Strum, "Parallel turbo coding interleavers: avoiding collisions in accesses to storage elements," IEEE Electron. Lett., vol. 38, no. 5, pp. 232-234, Feb. 2002.

[26] M. J. Thul, N. Wehn, and L. Rao, "Enabling high-speed turbo-decoding through concurrent interleaving," in Proc. IEEE Int. Symp. Circuits and Syst. (ISCAS), May 2002, pp. 897-900.

[27] F. Speziali and J. Zory, "Scalable and area efficient concurrent interleaver for high throughput turbo-decoders," in Proc. Euromicro Symp. Digit. Syst. Des. (DSD), Aug. 2004, pp. 334-341.

[28] A. Tarable, S. Benedetto, and G. Montorsi, "Mapping interleaving laws to parallel turbo and LDPC decoder architectures," IEEE Trans. Inf. Theory, vol. 50, no. 9, pp. 2002-2009, Sept. 2004.

[29] C. Berrou, Y. Saouter, C. Douillard, S. Kerouédan, and M. Jézéquel, "Designing good permutations for turbo codes: towards a single model," in Proc. IEEE Int. Conf. Commun. (ICC), June 2004, pp. 341-345.

[30] C. Neeb, M. J. Thul, and N. Wehn, "Network-on-chip-centric approach to interleaving in high throughput channel decoders," in Proc. IEEE Int. Symp. Circuits and Syst. (ISCAS), May 2005, pp. 1766-1769.

[31] O. Y. Takeshita, "On maximum contention-free interleavers and permutation polynomials over integer rings," IEEE Trans. Inf. Theory, vol. 52, no. 3, pp. 1249-1253, 2006.

[32] H. Moussa, O. Muller, A. Baghdadi, and M. Jezequel, "Butterfly and Benes-based on-chip communication networks for multiprocessor turbo decoding," in Proc. Des., Autom. \& Test in Europe (DATE), Apr. 2007, pp. $1-6$.

[33] A. Nimbalker, T. Blankenship, B. Classon, T. Fuja, and D. Costello, "Contention-free interleavers for high-throughput turbo decoding," IEEE Trans. Commun., vol. 56, no. 8, pp. 1258-1267, Aug. 2008.

[34] M. M. K. Eid M. Abdel-Hamid, Hossam A. H. Fahmy, "Memory conflict analysis for a multi-standard, reconfigurable turbo decoder," in Proc. IEEE Int. Symp. Circuits and Syst. (ISCAS), May 2011, pp. 2701-2704.

[35] A. Briki, C. Chavet, P. Coussy, and E. Martin, "A design approach dedicated to network-based and conflict-free parallel interleavers," in Proc. ACM Great Lakes Symp. on VLSI (GLSVLSI), May 2012, pp. $153-158$.

[36] E. Nieminen, "A contention-free parallel access by butterfly networks for turbo interleavers," IEEE Trans. Inf. Theory, vol. PP, no. 99, pp. 1-1, 2013.

[37] M.-C. Shin and I.-C. Park, "SIMD processor-based turbo decoder supporting multiple third-generation wireless standards," IEEE Trans. VLSI Syst., vol. 15, no. 7, pp. 801-810, 2007.

[38] J.-H. Kim and I.-C. Park, "A unified parallel radix-4 turbo decoder for mobile WiMAX and 3GPP-LTE," in Proc. IEEE Custom Integrated Circuits Conf. (CICC), Sept. 2009, pp. 487-490. 
[39] C.-H. Lin, C.-Y. Chen, E.-J. Chang, and A.-Y. Wu, "Reconfigurable parallel turbo decoder design for multiple high-mobility $4 \mathrm{G}$ systems," Springer J. Signal Process. Syst., vol. 73, no. 2, pp. 109-122, 2013.

[40] A. Vosoughi, G. Wang, H. Shen, J. R. Cavallaro, and Y. Guo, "Highly scalable on-the-fly interleaved address generation for UMTS/HSPA+ parallel turbo decoder," in Proc. IEEE Int. Conf. Appl.-Specific Syst., Archit. and Processors (ASAP), Jun. 2013, pp. 356-362.

[41] J. Ryu and O. Y. Takeshita, "On quadratic inverses for quadratic permutation polynomials over integer rings," IEEE Trans. Inf. Theory, vol. 52, no. 3, pp. 1254-1260, 2006.

[42] J. Lahtonen, J. Ryu, and E. Suvitie, "On the degree of the inverse of quadratic permutation polynomial interleavers," IEEE Trans. Inf. Theory, vol. 58, no. 6, pp. 3925-3932, 2012.

[43] J. Vogt and A. Finger, "Improving the max-log-MAP turbo decoder," IET Electronics letters, vol. 36, no. 23, pp. 1937-1939, 2000.

[44] H. Borrayo-Sandoval, R. Parra-Michel, L. Gonzalez-Perez, F. Printzen, and C. Feregrino-Uribe, "Design and implementation of a configurable interleaver/deinterleaver for turbo codes in 3GPP standard," in Proc. Int. Conf. Reconfig. Comput. and FPGAs, Dec. 2009, pp. 320-325.

[45] F. Kienle, N. Wehn, and H. Meyr, "On complexity, energy- and implementation-efficiency of channel decoders," IEEE Trans. on Commun., vol. 59, no. 12, pp. 3301-3310, Dec. 2011. 